\title{
Trimetazidine improves hepatic lipogenesis and steatosis in non-alcoholic fatty liver disease via AMPK-ChREBP pathway
}

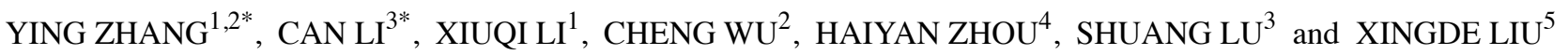 \\ ${ }^{1}$ Department of Cardiology, General Hospital of The Yangtze River Shipping, Wuhan, Hubei 430010; \\ Departments of ${ }^{2}$ Cardiology, ${ }^{3}$ Infectious Diseases and ${ }^{4}$ Clinical Research Centre, Affiliated Hospital of \\ Guizhou Medical University; ${ }^{5}$ Department of Cardiology, The Second Clinical Medical College of \\ Guiyang College of Chinese Medicine, Guiyang, Guizhou 550004, P.R. China
}

Received November 27, 2018; Accepted January 29, 2020

DOI: $10.3892 / \mathrm{mmr} .2020 .11309$

\begin{abstract}
Clinical studies have demonstrated that trimetazidine (TMZ) possesses a synergistic hypolipidemic effect together with statins, but the underlying mechanism remains to be elucidated. The present study aimed to investigate the role of TMZ in non-alcoholic fatty liver disease (NAFLD). By investigating the TMZ treatment of NAFLD, it was identified that high-fat diet (HFD) mice exhibit significant changes in several physiologic indices, including body weight, plasma lipids and glucose tolerance. Notably, hepatocyte bullous steatosis and fibrosis in HFD mice are greatly attenuated by 8 weeks of TMZ treatments. The results of the present study also indicated that the expression of carbohydrate-responsive element-binding protein (ChREBP), fatty acid synthase and acetyl-CoA carboxylase were all significantly reduced in the HFD + TMZ group compared with the HFD group. In order to confirm the hypothesis in vitro, the palmitate-treated liver cancer cell line (HepG2) was employed and similar results were obtained in TMZ-treated HepG2 cells. Furthermore, TMZ markedly upregulated the AMP-activated protein kinase (AMPK) signaling pathway and reduced the expression of forkhead box $\mathrm{O} 1$ (FOXO1) in the cells, while these effects controlled by TMZ were abolished by the AMPK inhibitor Compound $\mathrm{C}$. The present study reported that knockdown
\end{abstract}

Correspondence to: Dr Ying Zhang, Department of Cardiology, General Hospital of The Yangtze River Shipping, 5 Huiji Road, Wuhan, Hubei 430010, P.R. China

E-mail: zyts2008@126.com

Professor Xingde Liu, Department of Cardiology, The Second Clinical Medical College of Guiyang College of Chinese Medicine, 83 Feishan Road, Guiyang, Guizhou 550004, P.R. China

E-mail: 2360040895@qq.com

"Contributed equally

Key words: trimetazidine, non-alcoholic fatty liver disease, hepatic steatosis, AMP-activated protein kinase, carbohydrate-responsive element-binding protein, forkhead box $\mathrm{O} 1$ of FOXO1 expression by FOXO1 small interfering RNA resulted in a reduction of ChREBP protein expression and post-transcriptional activity. In summary, for the first time, to the best of the authors' knowledge, the present study revealed a novel role of TMZ in hepatic steatosis; TMZ ameliorated ChREBP-induced de novo lipogenesis by activating the AMPK-FOXO1 pathway.

\section{Introduction}

One of the common health issues both in developed and developing countries around the world is the increasing epidemic of obesity-related chronic diseases, including non-alcoholic fatty liver disease (NAFLD), type 2 diabetes (T2D) and cardiovascular disease, which results in high costs in terms of health-care expenditure and quality of life (1-3). In the Western world and Asia, the prevalence of NAFLD is estimated as $15-30 \%$ of the population $(4,5)$. NAFLD is described as a presence of non-alcoholic hepatic steatosis and liver tissue inflammation (6). Patients with NAFLD have an increase in liver fat (triglyceride; TG) accumulation and hepatic insulin resistance (7). In addition, NAFLD has been associated with the increased risks of T2D, cardiovascular disease and metabolic syndrome (1). However, the exact molecular mechanisms of the regulation remain unclear and effective drugs for the treatment of NAFLD have yet to be found.

Trimetazidine (TMZ) is known to protect against myocardial ischemia injury, which inhibits fatty acid $\beta$-oxidation and enhances glucose oxidation to optimize cardiac energy metabolism $(8,9)$. TMZ has been used in the treatment of coronary heart disease in China. In previous years, Chinese researchers have found that TMZ has a synergistic hypolipidemic effect with statins $(10,11)$. However, the detailed molecular mechanism of TMZ remains unclear.

AMP-activated protein kinase (AMPK), a crucial cellular senor of energy homeostasis, can influence metabolism and energy balance at the whole body level by regulating metabolic and non-metabolic processes $(12,13)$. The AMPK signal pathway is also closely related to NAFLD $(14,15)$. As AMPK activity is reduced by inflammation, obesity and diabetes, increasing AMPK activity has been regarded as a viable 
therapeutic strategy to improve NAFLD (16). Some studies suggest that the enhanced AMPK signaling may attenuate liver lipid accumulation and hepatic fibrosis in mice $(17,18)$. AMPK and AKT signaling pathways can increase forkhead box $\mathrm{O} 1$ (FOXO1) expression in a number of tissues and cells $(19,20)$.

The carbohydrate-responsive element-binding protein (ChREBP) has a crucial role in the regulation of hepatic lipogenesis, hepatic steatosis and insulin resistance $(21,22)$. ChREBP is a major mediator in glycolysis and lipogenesis via binding to the promoter region of lipogenic genes.

The present study aimed to investigate the effect of TMZ on NAFLD induced by a high-fat diet (HFD). It demonstrated that administration of TMZ alleviated hepatic steatosis and insulin resistance in HFD mice. The curative effects could be achieved by attenuating hepatic lipogenesis via the AMPK-ChREBP signaling pathway.

\section{Materials and methods}

Animal experiments and study design. The animal experiments were approved by the Institutional Animal Care and Research Advisory Committee at Guizhou Medicine University, Guiyang, China. A total of 18 8-week-old (weight, 18-20 g) male C57BL/6 mice (Experimental Animal Center of Hubei) were randomly divided and fed with a normal chow diet containing $4 \%$ fat (NCD group, $n=6$ ) or a HFD containing $60 \%$ fat and $2 \%$ cholesterol (HFD group $\mathrm{n}=6)(23)$, or a HFD plus daily infused TMZ (40 mg/kg) by gavage (HFD + TMZ group, $n=6)$ for 8 weeks before sacrifice. All mice were housed in a temperature-controlled environment in $12 / 12 \mathrm{~h}$ light/dark cycles with free access to food and water.

Cell culture and treatments. HepG2 cells were maintained in Dulbecco's modified Eagle's medium (DMEM, Sigma-Aldrich; Merck KGaA) with $10 \%$ fetal bovine serum (FBS, Beijing Transgen Biotech Co., Ltd.), in an incubator kept at $37^{\circ} \mathrm{C}$ with $5 \% \mathrm{CO}_{2}$. At $70-80 \%$ confluence, the cells were incubated in serum-free medium or serum-free medium containing $0.25 \mathrm{mM}$ palmitic acid (PA; Sigma-Aldrich; Merck KGaA), or $10 \mu \mathrm{M}$ TMZ (Laboratoires Servier) or $1 \mu \mathrm{M}$ Compound C (MedChemExpress) for another $24 \mathrm{~h}$.

Histological analysis. The livers were fixed in $4 \%$ formaldehyde at room temperature and embedded in paraffin, sections were at a thickness of $5 \mu \mathrm{m}$. All staining was performed at room temperature. The hepatic morphology was detected by haematoxylin and eosin (H\&E) staining and Oil Red O (ORO) staining. Staining with Sirius red was performed to visualize liver tissue fibrosis.

Biochemical parameters. TG and total cholesterol (TC) levels in plasma or HepG2 cells were determined by GRO-PAP analysis kits (Nanjing Jiancheng Bioengineering Institute). TG and TC in liver were measured on an AEROSET Clinical Chemistry System (Abbott Laboratories).

Small interfering RNA (siRNA). siRNA targeting FOXO1 (NM_002015) and the negative control (NC) were purchased from Guangzhou RiboBio Co., Ltd. siRNA transfections were performed with Lipofectamine ${ }^{\circledR} 2000$ (Invitrogen, Thermo Fisher Scientific, Inc.) at a final concentration of $100 \mathrm{nM}$. After $48 \mathrm{~h}$ cells were used for subsequent experiments.

$R N A$ extraction and reverse transcription-quantitative $(R T-q)$ $P C R$. Total RNA was isolated using a TRIzol reagent kit (Invitrogen, Thermo Fisher Scientific, Inc.) and quantified by qPCR using a SYBR Premix Ex Taq II mix (Takara Bio, Inc.), according to the manufacturer's instructions. The quantitative PCR was performed in an ABI 7900 Fast Real-Time PCR System (Applied Biosystems Inc.), following the manufacturer's protocols. mRNA levels were quantified using the $2^{-\Delta \Delta \mathrm{Cq}}$ method (24) and normalized to the internal gene $\beta$-actin, primers are listed in Table I.

Western blot analysis. Protein was extracted using RIPA lysis buffer (Wuhan Boster Biological Technology, Ltd.). Protein $(20 \mu \mathrm{g})$ was separated by $8 \%$ SDS-PAGE and transferred on to PVDF membrane. The membranes were incubated with antibodies of ChREBP (1:1,000, Abcam, cat. no. ab92809), fatty acid synthase (FASN; 1:500, Wuhan Boster Biological Technology, Ltd., cat. no. PB0909), acetyl-CoA carboxylase (ACC; 1:500, Wuhan Boster Biological Technology, Ltd., cat. no. BM4414), AMPK (1:1,000, Cell Signaling Technology, Inc., cat. no. 2532), phosphorylated (p)-AMPK (Thr172; 1:1,000, Cell Signaling Technology, Inc., cat. no. 2535), AKT (1:1,000, Cell Signaling Technology, Inc., cat. no. 9272), p-AKT (Ser473; 1:1,000, Cell Signaling Technology, Inc., cat. no. 9271), GAPDH (1:2,000, Wuhan Boster Biological Technology, Ltd., cat. no. BM1623) and FOXO1 (1:500, Wuhan Boster Biological Technology, Ltd., BM4249) overnight at $4^{\circ} \mathrm{C}$. Then, membranes were incubated with rabbit anti-mouse $\operatorname{lgG}$ (cat. no. BA1048) and goat anti-rabbit $\operatorname{lgG}$ antibodies (cat. no. BA1039; both from Wuhan Boster Biological Technology, Ltd.). Finally, the results were detected by ECL reagents (Beyotime Institute of Biotechnology) and semi-quantified by densitometry using the Tanon 5200 automatic chemiluminescent imaging system (Tanon Science and Technology Co., Ltd).

Cholesterol efflux. The HepG2 cells were subjected to DMEM with $5 \mathrm{nM}$ 22-NBD cholesterol (Invitrogen; Thermo Fisher Scientific, Inc.) for $6 \mathrm{~h}$. The cholesterol efflux was measured following 2-h incubation with apolipoprotein (apo)A-I $(25 \mathrm{mg} / \mathrm{ml})$ or high-density lipoprotein (HDL; Guangzhou Yiyuan Biotech Co., Ltd.) particles isolated from human plasma $(50 \mathrm{mg} / \mathrm{ml})$.

Luciferase assay. Bioinformatics analyses (http:///jaspar. genereg.net/) demonstrated that there were a number of FOXO1 binding sites in the ChREBP promoter region. The 2,256 bp ChREBP promoter sequence was amplified from human DNA by PCR, with Mlu1 or HindIII restriction enzyme cutting site at $5^{\prime}$ and $3^{\prime}$ end, respectively. The primers used were 5'-CTCTGAACCCATCTTTGAGGC-3' and 5'-AGTCTGTGTCCGAGTCCGAgT-3'. PCR products were digested and inserted into a pGL-3 luciferase reporter vector (Beijing Augct DNA-Syn Biotechnology Co., Ltd.). 293T cells (1x10 $10^{6}$ cells per well) in 24 -well plates were co-transfected with $400 \mathrm{ng}$ of plasmid, $50 \mathrm{ng}$ of Renilla luciferase 
Table I. Primer sequences for PCR.

\begin{tabular}{|c|c|c|}
\hline Gene name & Genera & Primer sequences $\left(5^{\prime}-3^{\prime}\right)$ \\
\hline ChREBPa & Mouse & $\begin{array}{l}\text { F: CGACACTCACCCACCTCTTC } \\
\text { R: TTGTTCAGCCGGATCTTGTC }\end{array}$ \\
\hline $\mathrm{ACC}$ & Mouse & $\begin{array}{l}\text { F: CTTCGGGGTGGTTCTTGGGTTGTG } \\
\text { R: CCTGCATCCGGCCTGGTGTG }\end{array}$ \\
\hline FASN & Mouse & $\begin{array}{l}\text { F: ATCCTGGAACGAGAACACGATCT } \\
\text { R: AGAGACGTGTCACTCCTGGACTT }\end{array}$ \\
\hline TGF- $\beta$ & Mouse & $\begin{array}{l}\text { F: GCAGTGGCTGAACCAAGGA } \\
\text { R: AGCAGTGAGCGCTGAATCG }\end{array}$ \\
\hline Collagen IV & Mouse & $\begin{array}{l}\text { F: CAACCTGGACGCCATCAAG } \\
\text { R: CAGACGGCTGAGTAGGGAACA }\end{array}$ \\
\hline АСТВ & Mouse & $\begin{array}{l}\text { F: CAGCCTTCCTTCTTGGGTAT } \\
\text { R: TGGCATAGAGGTCTTTACGG }\end{array}$ \\
\hline ChREBP & Human & $\begin{array}{l}\text { F: AGTGCTTGAGCCTGGCCTAC } \\
\text { R: TTGTTCAGGCGGATCTTGTC }\end{array}$ \\
\hline $\mathrm{LXR} \alpha$ & Human & $\begin{array}{l}\text { F: AGAAGAACAGATCCGCCTGAAG } \\
\text { R: GGCAAGGATGTGGCATGAG }\end{array}$ \\
\hline SREBP1 & Human & $\begin{array}{l}\text { F: GGGCGTGAAGACTGAGGTG } \\
\text { R: CTGCTGCCAAGGGACAAG }\end{array}$ \\
\hline TRAK2 & Human & $\begin{array}{l}\text { F: ATGAGTCAATCCCAGAATGCAA } \\
\text { R: TCAGTCCTCCTTCAGGACACC }\end{array}$ \\
\hline ABCA1 & Human & $\begin{array}{l}\text { F: TACAGCCAGAAAGACACCAG } \\
\text { R: CACAGTAGACTTTGGGAGAG }\end{array}$ \\
\hline ABCG1 & Human & $\begin{array}{l}\text { F: TCGGTGGATGAGGTGGTG } \\
\text { R: TGGGCTTCCGTGAGGTTA }\end{array}$ \\
\hline PKLR & Human & $\begin{array}{l}\text { F: GTGGACATCGTCTTTGCCT } \\
\text { R: TCTTGATGCCGTGTCCTTC }\end{array}$ \\
\hline FASN & Human & $\begin{array}{l}\text { F: CGCTCGGCATGGCTATCT } \\
\text { R: CTCGTTGAAGAACGCATCCA }\end{array}$ \\
\hline $\mathrm{ACC}$ & Human & $\begin{array}{l}\text { F: GGATGGTGTTCACTCGGTAATAG } \\
\text { R: GGGTGATATGTGCTGCGTCAT }\end{array}$ \\
\hline АСТВ & Human & $\begin{array}{l}\text { F: TGGATCAGCAAGCAGGAGTATG } \\
\text { R: GCATTTGCGGTGGACGAT }\end{array}$ \\
\hline
\end{tabular}

chREBP, carbohydrate-responsive element-binding protein; ACC, acetyl-CoA carboxylase; FASN, fatty acid synthase; TGF- $\beta$, transforming growth factor- $\beta$; ACTB, $\beta$-actin; LXRa, liver X receptor $\alpha$; SREBP1, sterol regulatory element-binding 1 ; TRAK2, trafficking kinesin protein 2; ABCA1, ATP-binding cassette subfamily A member 1; ABCG1, ATP-binding cassette subfamily G member 1; PKLR, pyruvate kinase L/R.

plasmid and $100 \mathrm{nM}$ si-FOXO1 or $100 \mathrm{nM}$ si-NC, using Lipofectamine ${ }^{\circledR} 2000$ (Invitrogen, Thermo Fisher Scientific, Inc.). Cells were incubated at $37^{\circ} \mathrm{C}$ and $5 \% \mathrm{CO}_{2}$. After $48 \mathrm{~h}$, the cells were washed using cold PBS and lysed with passive lysis buffer (Promega Corporation). Luciferase activities were then measured by a luminometer (Sirius; Berthold Detection Systems $\mathrm{GmbH}$ ) according to the manufacturer's instructions. Luciferase expression levels were adjusted with reference to Renilla luciferase activity. Six independent experiments were performed for each reporter vector.

Statistical analysis. Statistical analyses were performed with SPSS 24.0 (IBM Corp.). Quantitative variables were expressed as mean \pm standard deviation of $n$ experiments. Comparisons of quantitative variables between two groups were performed by the paired or unpaired Student's t-test. One-way ANOVA was used to compare multiple variables followed by a Tukey's post hoc test. The differences among three or more groups were analyzed by a multiple comparisons test. All probability values were two-sided and $\mathrm{P}<0.05$ was considered to indicate a statistically significant difference.

\section{Results}

TMZ attenuates symptoms in HFD mice. Metabolic disorder was induced in mice with continuous HFD exhibiting body weight gain, hyperlipidemia, hyperglycemia and glucose intolerance compared with NCD mice (Fig. 1). To clarify the potential effect of TMZ on HFD-mediated metabolic disorder, the administration of TMZ by gavage was used. The TMZ significantly decreased the liver weight of HFD + TMZ mice compared with the HFD mice, but it did not change the whole 


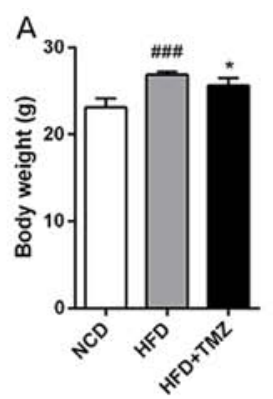

B
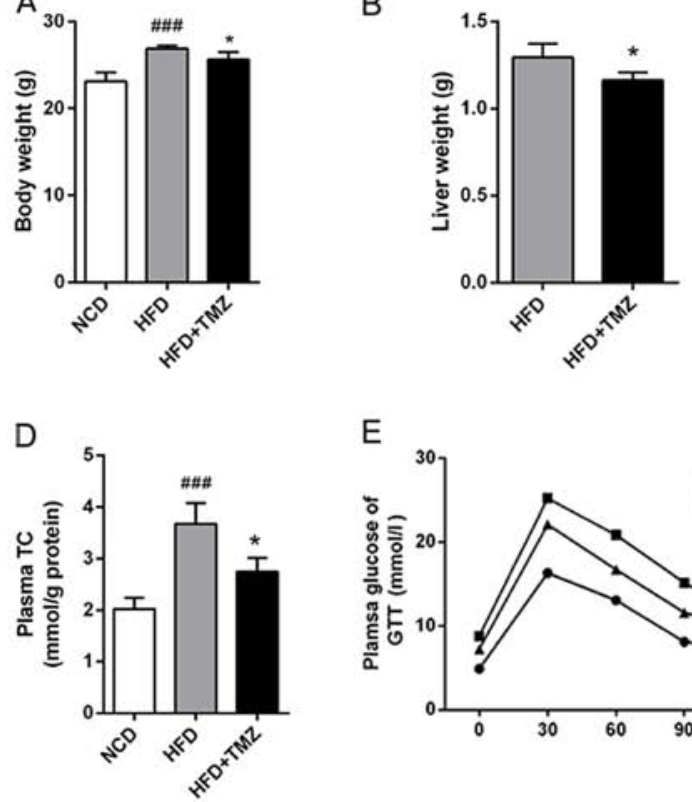

$\mathrm{E}$

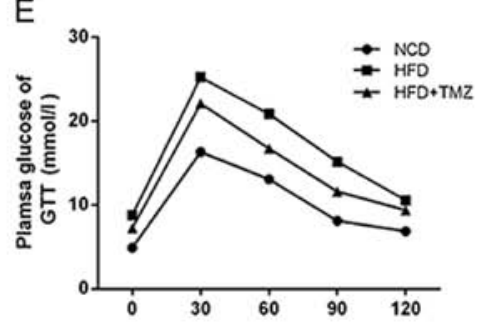

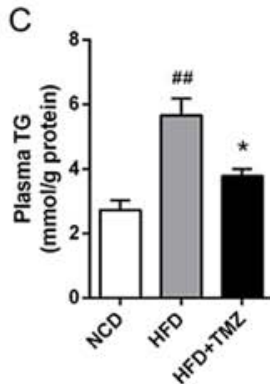

$\mathrm{F}$

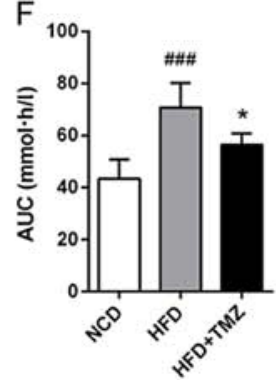

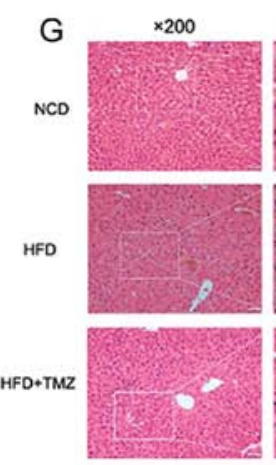

Zoomed
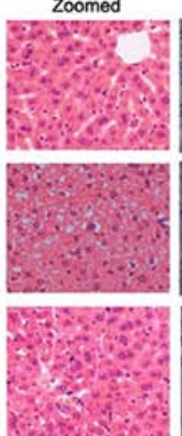

$\mathrm{H}$

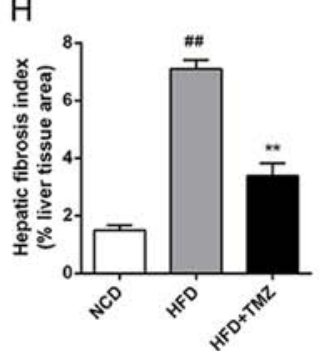

$\times 200$
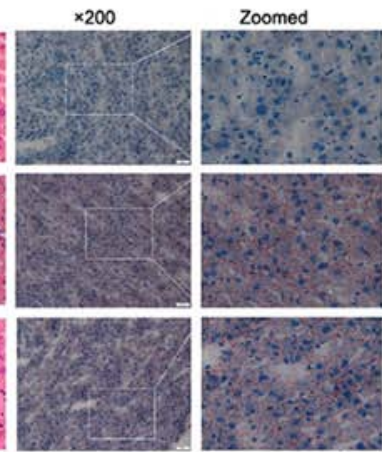
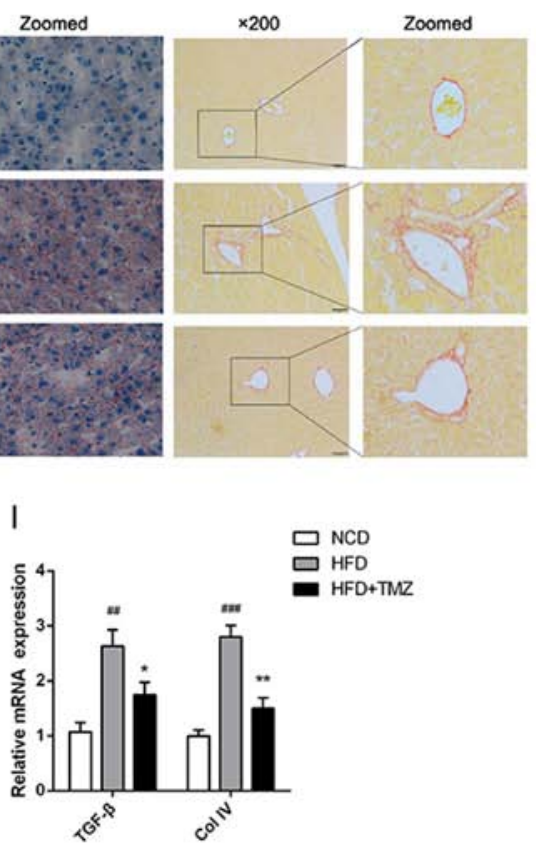

Figure 1. TMZ improved metabolic characteristics and hepatic fibrosis in treated mice. The C57BL/6J mice were fed with a NCD, HFD or HFD + daily infused TMZ (40 mg/kg) by gavage (HFD + TMZ) for 8 weeks (n=6). (A and B) Body weight and liver weight are shown. (C and D) Mice plasma TG and TC were detected by the GRO-PAP method. (E and F) After an overnight, fast and serial tail blood glucose was measured before and after glucose administration ( $2 \mathrm{~g} / \mathrm{kg}$, intraperitoneal injection). (G) Representative images of H\&E staining, Oil Red O staining and Sirius red staining of mouse livers (magnification, x 200). (H) Hepatic fibrosis index. (I) Relative mRNA levels of TGF- $\beta$ and Col IV. $\left({ }^{\#} \mathrm{P}<0.01\right.$ vs. NCD group, ${ }^{\# \# ~} \mathrm{P}<0.001$ vs. NCD group, ${ }^{*}<0.05$ vs. HFD group, ${ }^{* *} \mathrm{P}<0.05$ vs. HFD group). TMZ, trimetazidine; NCD, normal chow diet; HFD, high fat diet; TG, triglyceride; TC, total cholesterol; GTT, glucose tolerance test; TGF- $\beta$, transforming growth factor- $\beta$; Col IV, Collagen I.

body weight as shown in Fig. 1A and B. HFD + TMZ mice exhibited $25-30 \%$ lower plasma TG/TC levels than those of HFD mice (Fig. 1C and D) and improved glucose tolerance compared with the HFD mice (Fig. 1E and F). All these data led to the hypothesis that TMZ might ameliorate hepatic lipid metabolism in HFD mice.

TMZ ameliorates hepatic fibrosis in vivo. To investigate the hypothesis above, the liver was investigated. As shown in Fig. 1G, H\&E and ORO staining demonstrated excessive lipid accumulation in HFD mice liver compared with the NCD mice liver. TMZ markedly decreased hepatocyte bullous steatosis and hepatic fibrosis in HFD + TMZ mice liver compared with the HFD mice (Fig. $1 \mathrm{G}$ and $\mathrm{H}$ ). In addition, TMZ decreased the expression of transforming growth factor- $\beta$ and Collagen IV in livers of HFD + TMZ mice compared with those of the HFD + TMZ mice, which also suggested that TMZ protected hepatic fibrosis in the livers of HFD + TMZ mice compared with those of the HFD + TMZ mice (Fig. 1I). 

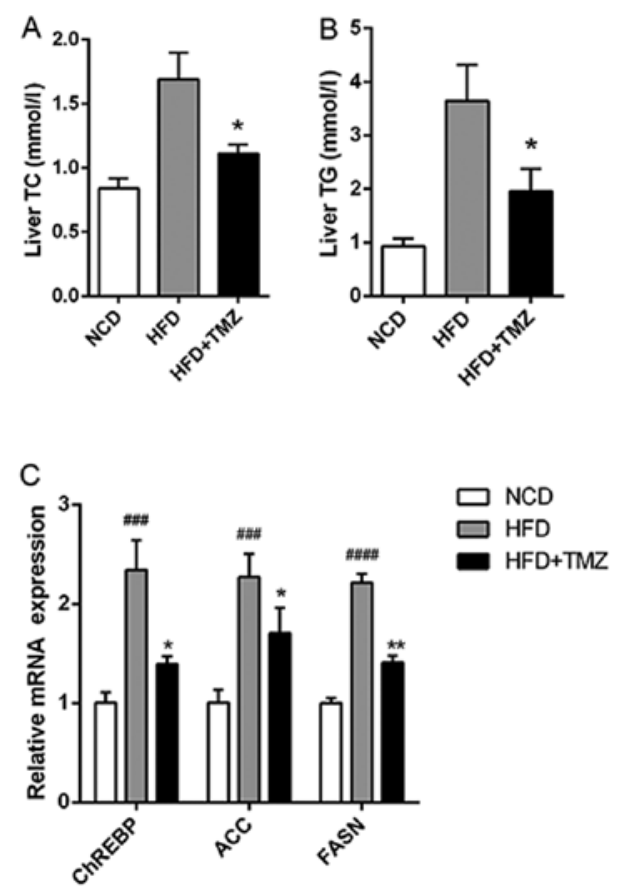

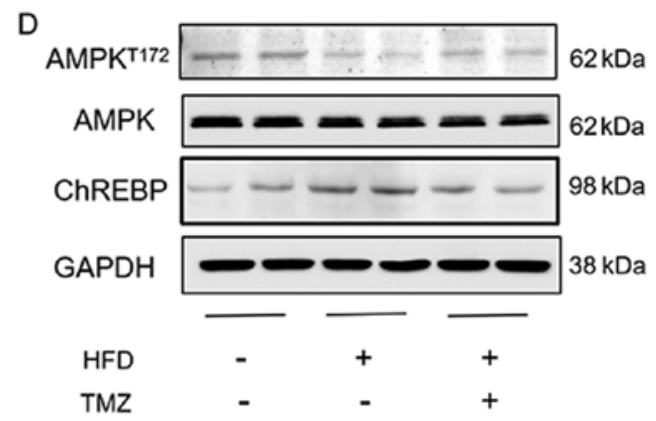

E

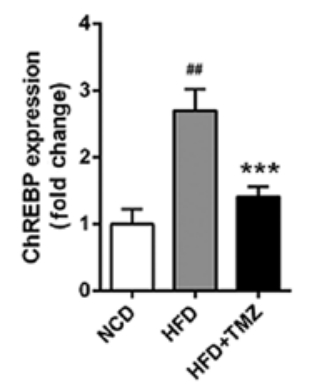

Figure 2. TMZ attenuated hepatic lipid accumulation and lipogenic gene expression in vivo. (A and B) Hepatic TG and TC in treated mice. (C) Relative $m R N A$ levels of ChREBP, ACC and FASN in liver of treated mice. (D-F) Protein levels of ChREBP and AMPK in liver of treated mice. $\left({ }^{*} \mathrm{P}<0.05\right.$ vs. NCD group,

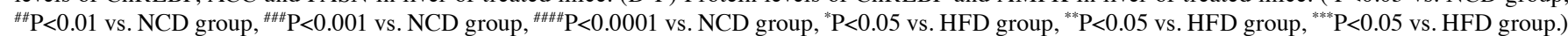
TMZ, trimetazidine; NCD, normal chow diet; HFD, high fat diet; TG, triglyceride; TC, total cholesterol; ChREBP, carbohydrate-responsive element-binding protein; ACC, acetyl-CoA carboxylase; FASN, fatty acid synthase; AMPK, AMP-activated protein kinase.

TMZ ameliorates hepatic lipid synthesis and suppressed $H F D$-related activation of AMPK in vivo. The hepatic TG/TC level in HFD + TMZ mice was markedly decreased compared with HFD mice (Fig. 2A and B). Notably, TMZ treatment demonstrated a lower mRNA and protein expression level of ChREBP, which was a critical transcription factor in hepatic lipogenesis in the liver of HFD + TMZ mice compared with those of the HFD mice (Fig. 2C and D). As expected, TMZ had the same effects on mRNA expression of ACC and FASN in livers of HFD + TMZ mice compared with those of the HFD mice. Furthermore, HFD mice demonstrated decreased phosphorylation of AMPK (Thr172) compared with NCD mice (Fig. 2D). TMZ treatment significantly reversed the reduced phosphorylation of AMPK.

$T M Z$ reduces the lipogenic gene expression in vitro. To verify the protective action of TMZ on NAFLD in vitro, the palmitate-treated liver cancer cell line HepG2 was used to confirm the animal experiment results. As demonstrated in Fig. 3A and B, ORO staining demonstrated that TMZ reduced excessive lipid accumulation, as before. There was no evidence to show that TMZ effected cholesterol efflux to apoA-I and HDL in liver cancer cell lines (Fig. 3C). A number of fat synthesis-related genes and cholesterol efflux-related genes were detected in liver cancer cells treated with TMZ and palmitate. TMZ only decreased the ChREBP mRNA expression. Liver $X$ receptor (LXR) $\alpha$, sterol regulatory element-binding 1 (SREBP1), CD36, SCARB1, trafficking kinesin protein 2, ATP-binding cassette subfamily A member 1 and ATP-binding cassette subfamily $\mathrm{G}$ member 1 demonstrated no significant difference in expression (Fig. 3D). In addition, TMZ also significantly decreased the mRNA and protein expression of key lipogenic genes, such as pyruvate kinase $\mathrm{L} / \mathrm{R}, \mathrm{ACC}$ and FASN (Fig. 3E and G).

TMZ alleviates hepatic lipogenesis via AMPK-FOXOI pathway. It was further investigated how TMZ affected fatty acid and fat synthesis in the liver cancer cell line. The level of TG and cholesterol in HepG2 cells are shown in Fig. 4A and B. The reduced TG and cholesterol level in TMZ-treated liver cancer cells were both increased following intervention with the AMPK inhibitor Compound C. It was then established that TMZ increased the PA-related activation of AMPK and suppressed the PA-related activation of AKT in vitro. All these effects were reversed when the Compound $\mathrm{C}$ was added (Fig. 4C-E). The expression of FOXO1 was associated with the AMPK/AKT pathway (Fig. 4F). Nevertheless, the relationship between the AMPK/AKT pathway and ChREBP-mediated lipid accumulation remains to be determined. As shown in Fig. 4G and $\mathrm{H}$, suppression of FOXO1 decreased the expression of ChREBP in the HepG2 and TMZ-PA-treated HepG2 cells. Furthermore, the suppression of FOXO1 decreased the luciferase reporter activity of the vector in 293T cells (Fig. 4I). These results suggested that TMZ suppressed hepatic lipogenesis by regulating ChREBP expression via AMPK-FOXO1 pathway.

\section{Discussion}

The present study conducted a mouse model mimicking human NAFLD with continuous HFD. The HFD mice demonstrated obesity, hyperlipidemia, hyperglycemia and glucose intolerance. The TMZ treatment alleviated the 
A

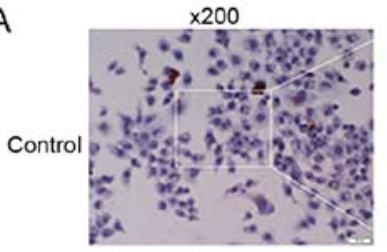

PA

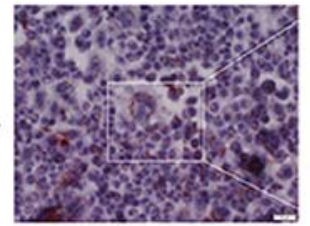

$\mathrm{PA}+\mathrm{TMZ}$

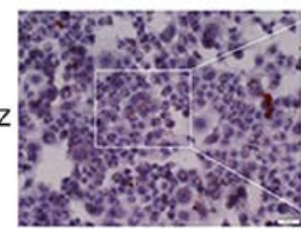

Zoomed
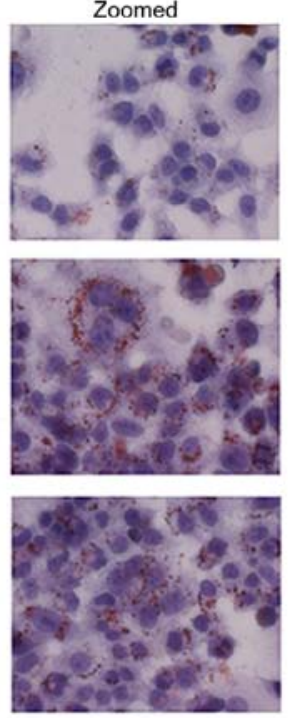

B

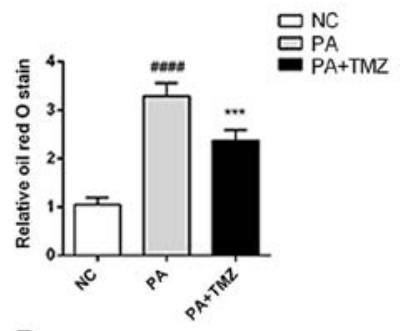

C

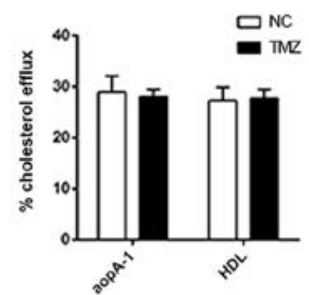

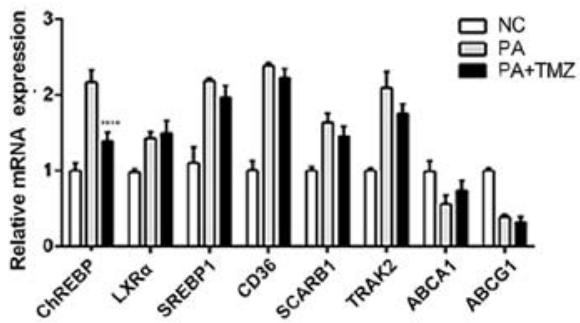

E

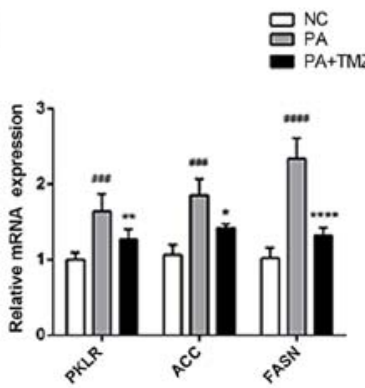

F

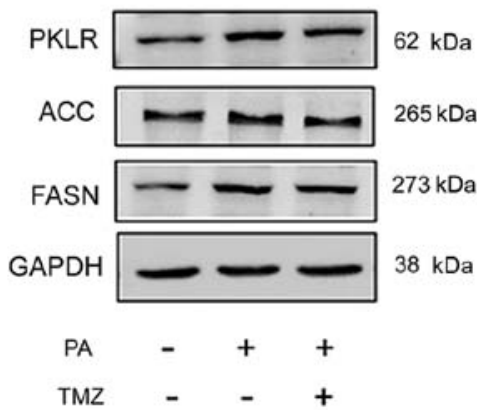

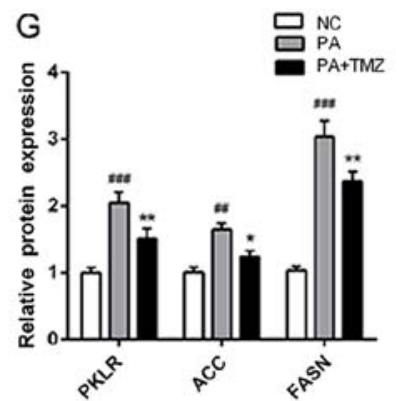

Figure 3. TMZ attenuated hepatic lipid accumulation and lipogenic gene in vitro. (A) Histopathological examination of palmitate-treated HepG2 cells with Oil Red O staining (x200). (B) Hepatic fibrosis index. (C) Cholesterol efflux to apoA-I and HDL. (D) Relative mRNA levels of key transcription factors in lipogenesis and other cholesterol efflux regulatory protein. (E) Relative mRNA and (F and G) protein levels of PKLR, ACC and FASN in HepG2 cells.

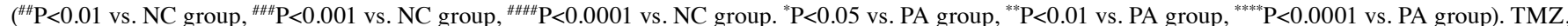
trimetazidine; apoA-I, apolipoprotein A1; HDL, high-density lipoprotein; PKLR, pyruvate kinase L/R; ACC, acetyl-CoA carboxylase; FASN, fatty acid synthase; PA, palmitic acid; NC, negative control.

clinical phenotypes and pathophysiological changes, including liver weight gain, hyperlipidemia, glucose intolerance and hepatic steatosis. Furthermore, TMZ also demonstrated the same effects in palmitate-treated HepG2 cells. These effects were associated with the reduction of hepatic lipogenesis. TMZ restrained the downregulation of phosphorylation of AMPK (Thr172) and AKT (Ser473) led by PA, downregulated ChREBP and ChREBP downstream genes and reduced the synthesis of lipids. In addition, the AMPK/AKT pathway inhibited FOXO1 expression. Furthermore, the suppression of FOXO1 was involved in the downregulation via decrease in the transcriptional activity of ChREBP promoter region.

The deposition of lipid droplets in the cells of the liver (that is, hepatic steatosis) is the hallmark of NAFLD. This deposition is strongly associated with the increasing fat synthesis. Up to $30 \%$ of patients with NAFLD develop non-alcoholic steatohepatitis (NASH), which depends on the presence of inflammation, oxidative stress and injury to liver cells, and a patient with NASH may progress to developing liver cancer (25). In human and mouse models, NAFLD increases the risk of liver cancer (26). It is important to understand the mechanisms of NAFLD and develop new intervention strategies (27).

TMZ has already been widely used in the treatment of stable angina pectoris, where it mobilizes myocardial fatty acid oxidation and glucose metabolism (11). A previous study suggests that TMZ can change energy production from fatty acid oxidation to glucose oxidation by inhibiting the enzymatic activity of long-chain 3-ketoacyl CoA thiolase and increasing the activity of pyruvate dehydrogenase to facilitate the transformation of pyruvate to acetyl Co-A (28). Chen et al (29) demonstrate that pressure overload-induced early cardiac energy dysfunction is ameliorated with the treatment of TMZ at $40 \mathrm{mg} / \mathrm{kg} / \mathrm{day}$. Although TMZ has been regarded as a metabolic medicine, with a synergistic hypolipidemic effect with statins, the effect of TMZ on lipid metabolism remains to be elucidated.

The present study, for the first time to the best of the authors' knowledge, established the relationship between TMZ and NAFLD. HFD mouse model or palmitate-treated cell model demonstrated TG accumulation. ChREBP plays important roles in modulating the process of glycolysis and 

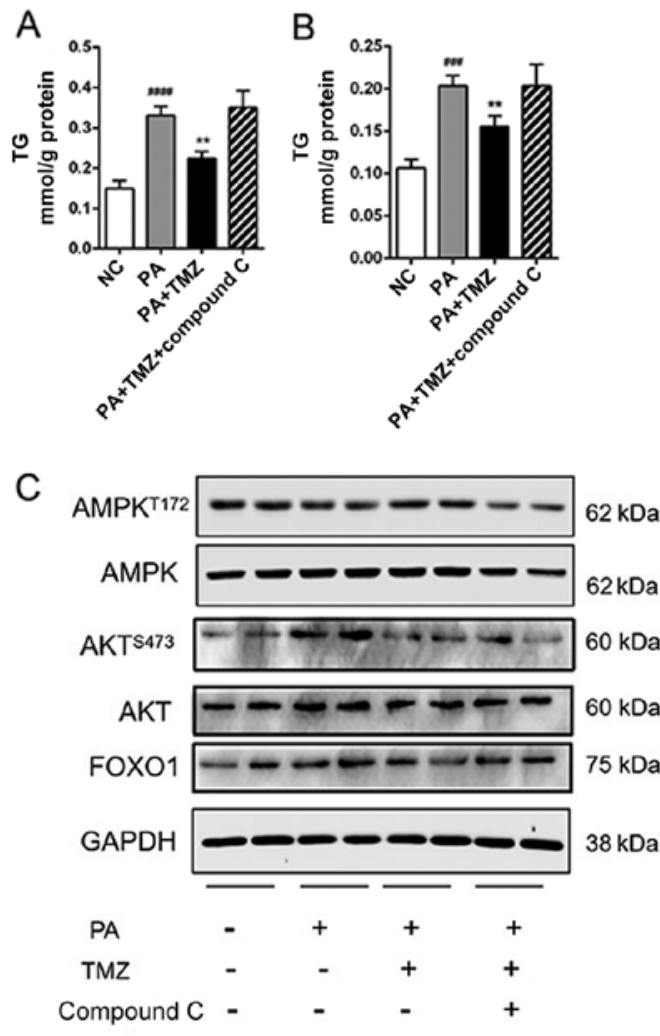
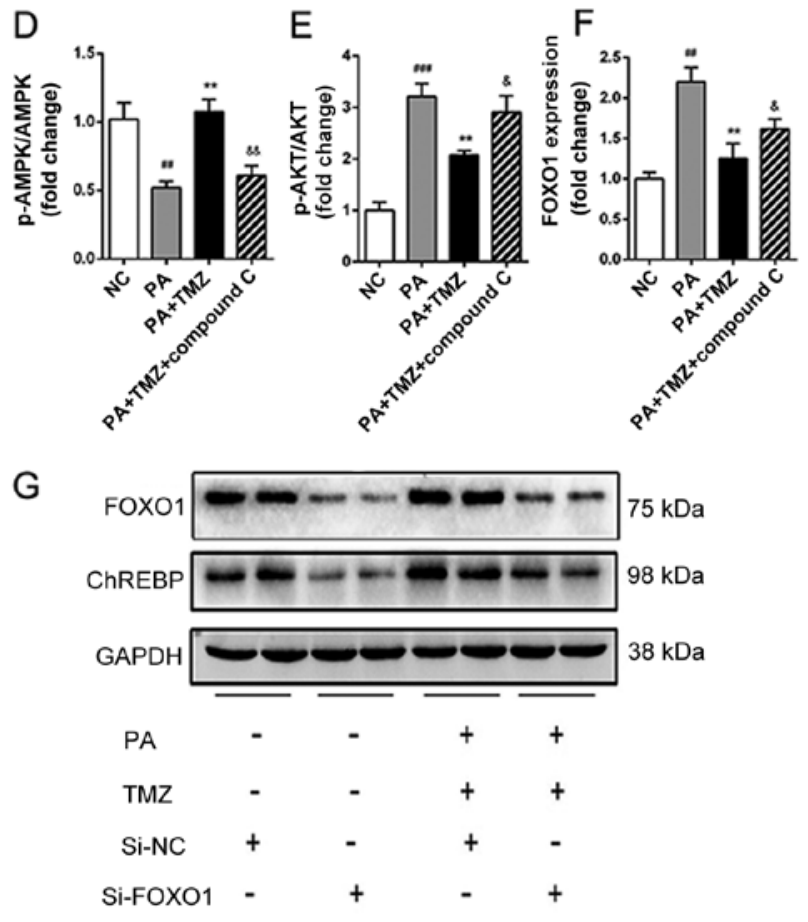

$\mathrm{H}$

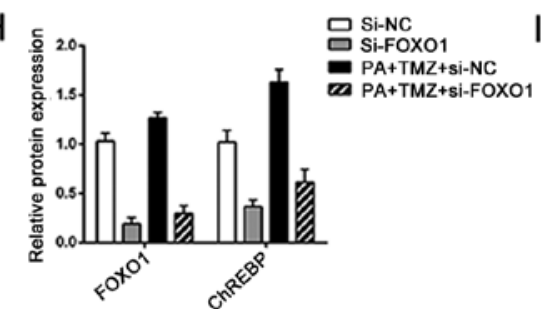

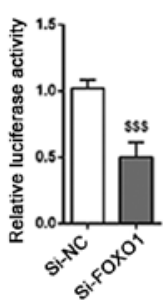

Figure 4. TMZ reduced hepatic de novo lipogenesis by activating AMPK-FOXO1-ChREBP signaling pathway in vitro. (A and B) TG and TC accumulation in HepG2 cells. (C-F) Protein levels of p-AMPK, AMPK, p-AKT, AKT and FOXO1 in HepG2 cells. (G and H) Protein levels of FOXO1 and ChREBP in

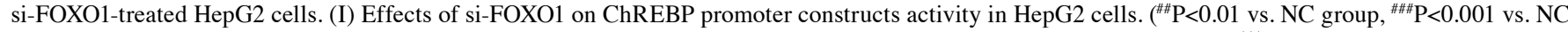

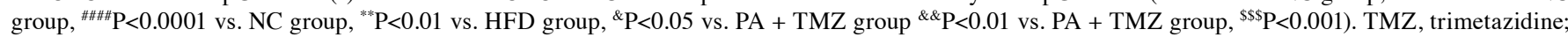
FOXO1, forkhead box O1; chREBP, carbohydrate-responsive element-binding protein; TG, triglyceride; TC, total cholesterol; p-, phosphorylated; si-, small interfering RNA; NC, negative control; HFD, high fat diet; PA, palmitic acid; AMPK, AMP-activated protein kinase; NCD, normal chow diet.

lipogenesis (30). Some key transcription factors, including PPAR, LXR, RXR, SREBP1 and ChREBP, link the molecular and metabolic bases of fatty acid regulation to NAFLD (31). ChREBP expression is positively related to the degree of hepatic steatosis in human and mice with NAFLD (32). Our previous study also confirmed the decreased expression of ChREBP attenuated lipid synthesis and accumulation (33). As expected, the HFD mouse demonstrated increased ChREBP-mediated fat syntheses. In addition, the model also demonstrated decreased phosphorylation of AMPK (Thr172). The TG accumulation and liver steatosis were alleviated with the addition of TMZ in a $40 \mathrm{mg} / \mathrm{kg} / \mathrm{day}$ dose in vivo and $10 \mu \mathrm{M}$ in vitro as in the experimental design of Chen et al (29). The reduced phosphorylation of AMPK was increased by TMZ. The ChREBP expression was also influenced by the drug, but the other vital transcription factors in lipogenesis and other cholesterol efflux regulatory protein expression were not affected. The present study suggested that the ChREBP gene and the AMPK pathway might be involved in this novel role for TMZ.

The AMPK inhibitor, Compound $\mathrm{C}$, was used to verify the connection between ChREBP and AMPK in vitro. As expected, Compound $\mathrm{C}$ inhibited the phosphorylation of AMPK and AKT. The inhibitor also alleviated the protective effect of TMZ in palmitate-treated HepG2 cells. These observations corroborated the hypothesis that $\mathrm{TMZ}$ ameliorates hepatic steatosis via the AMPK/AKT pathway. Furthermore, the present study reported that the AMPK/AKT pathway altered FOXO1 expression, which plays an important role in the processes in NAFLD (34). The transcription factor FOXO1 is the main target of insulin signaling, and contributes to the regulation of lipid and glucose metabolism (35). Bioinformatics analyses (http:///jaspar.genereg.net/) demonstrated that there were a number of FOXO1 binding sites in the ChREBP promoter region. As expected, the suppression of FOXO1 resulted in a reduction of ChREBP protein expression and post-transcriptional activity. The present study confirmed the relationship between the AMPK/AKT pathway and NAFLD, and demonstrated that TMZ ameliorated ChREBP-induced de novo lipogenesis by activating AMPK-FOXO1 signaling.

The present study has several limitations with a few factors uninterpreted. As mentioned above, the experiments were designed to test the role of TMZ in disease. Thus the role of TMZ in a control group was not tested, which led to 
the lack of a sham + TMZ group. It was observed that the liver weight of NAFLD was significantly attenuated by TMZ, but due to some unavoidable factors, the original data of normal liver weight was lost. The function of the AMPK pathway was also not confirmed in vivo. TMZ treatment on NAFLD should have been confirmed by combination therapy with the Compound $\mathrm{C}$ or AMPK gene deficiency mice. Another limitation was the mechanism of the regulation of AMPK by TMZ, which requires further experiments. Furthermore, the cell line used (HepG2) is a problematic cell line, it was originally identified as a hepatocellular carcinoma cell line, however it has since been shown to be from a hepatoblastoma (PubMed =19751877).

In summary, the present study reveals a novel role for TMZ: Protecting hepatic steatosis in NAFLD. The protective action of TMZ depends on its ability to reduce ChREBP-mediated de novo lipogenesis via the AMPK/AKT/FOXO1 signaling pathway. The findings of the present study show that TMZ may provide potentially therapeutic strategies for NAFLD.

\section{Acknowledgements}

The authors would like to thank Dr Jin Li (Tsinghua University, Beijing, China) for his English editing of the manuscript.

\section{Funding}

This work was supported by the National Natural Science Foundation of China (grant no. 31760294), the Science and Technology Fund of Guizhou Provincial Health Department [grant nos. 31760294 qiankehejichu (2016) 1120, qiankehejichu (2018) 1137)], and the Fund of Guiyang Science and Technology department [grant nos. (2017) 5-14, (2017) 30-10], the Health and Family Planning Commission of Guizhou Province (grant no. gzwjkj2017-1-016), the Fund of Qiannan Science and Technology department [grant no. qiannankeheshezi (2017) 73].

\section{Availability of data and materials}

The datasets used and/or analyzed during the current study are available from the corresponding author on reasonable request.

\section{Authors' contributions}

YZ participated in the research design, performed experiments, conducted statistical analyses and drafted the manuscript. CL performed experiments, performed statistical analyses and drafted the manuscript. CW performed the animal experiments. HZ performed statistical analyses. SL performed statistical analyses and edited the final manuscript. XDL conceived the study and edited the final manuscript. All authors read and approved the final manuscript.

\section{Ethics approval and consent to participate}

The animal experiments were approved by the Institutional Animal Care and Research Advisory Committee at Guizhou Medicine University, Guiyang, China.

\section{Patient consent for publication}

Not applicable.

\section{Competing interests}

The authors declare that they have no competing interests.

\section{References}

1. Yki-Järvinen H: Non-alcoholic fatty liver disease as a cause and a consequence of metabolic syndrome. Lancet Diabetes Endocrinol 2: 901-910, 2014.

2. Malik VS, Willett WC and Hu FB: Global obesity: Trends, risk factors and policy implications. Nat Rev Endocrinol 9: 13-27, 2013.

3. Alberti KGMM, Eckel RH, Grundy SM, Zimmet PZ Cleeman JI, Donato KA, Fruchart J-C, James WPT, Loria CM and Smith SC Jr; International Diabetes Federation Task Force on Epidemiology and Prevention; National Heart, Lung, and Blood Institute; American Heart Association; World Heart Federation; International Atherosclerosis Society; International Association for the Study of Obesity: Harmonizing the metabolic syndrome: A joint interim statement of the International Diabetes Federation Task Force on Epidemiology and Prevention; National Heart, Lung, and Blood Institute; American Heart Association; World Heart Federation; International Atherosclerosis Society; and International Association for the Study of Obesity. Circulation 120: 1640-1645, 2009.

4. Wong VW-S: Nonalcoholic fatty liver disease in Asia: A story of growth. J Gastroenterol Hepatol 28: 18-23, 2013.

5. Lazo M, Hernaez R, Eberhardt MS, Bonekamp S, Kamel I, Guallar E, Koteish A, Brancati FL and Clark JM: Prevalence of nonalcoholic fatty liver disease in the United States: The Third National Health and Nutrition Examination Survey, 1988-1994. Am J Epidemiol 178: 38-45, 2013.

6. Marengo A, Jouness RIK and Bugianesi E: Progression and natural history of nonalcoholic fatty liver disease in adults. Clin Liver Dis 20: 313-324, 2016.

7. Chalasani N, Younossi Z, Lavine JE, Diehl AM, Brunt EM, Cusi K, Charlton M and Sanyal AJ; American Gastroenterological Association; American Association for the Study of Liver Diseases; American College of Gastroenterologyh: The diagnosis and management of non-alcoholic fatty liver disease: Practice guideline by the American Gastroenterological Association, American Association for the Study of Liver Diseases, and American College of Gastroenterology. Gastroenterology 142: 1592-1609, 2012.

8. Jaswal JS, Keung W, Wang W, Ussher JR and Lopaschuk GD: Targeting fatty acid and carbohydrate oxidation--a novel therapeutic intervention in the ischemic and failing heart. Biochim Biophys Acta 1813: 1333-1350, 2011.

9. Lopaschuk GD, Barr R, Thomas PD and Dyck JRB: Beneficial effects of trimetazidine in ex vivo working ischemic hearts are due to a stimulation of glucose oxidation secondary to inhibition of long-chain 3-ketoacyl coenzyme a thiolase. Circ Res 93: e33-e37, 2003.

10. Chen $\mathrm{H}$ and $\mathrm{Xu} \mathrm{Y}$ : Clinical trial of trimetazidine combined with atorvastatin calcium in the treatment of coronary heart disease and angina pectoris with dyslipidemia. Zhongguo Lin Chuang Yao Li Xue Za Zhi 32: 966-968, 2016. (Chinese).

11. Zhou $X$ and Huang H: Therapeutic effect of atorvastatin combined trimetazidine on blood lipids,inflammatory factors and cardiac function in patients with coronary heart disease. Chin J Cardiovasc Rehabil Med 25: 276-279, 2016. (Chinese).

12. Hardie DG, Ross FA and Hawley SA: AMPK: A nutrient and energy sensor that maintains energy homeostasis. Nat Rev Mol Cell Biol 13: 251-262, 2012.

13. Hardie DG: AMPK: Positive and negative regulation, and its role in whole-body energy homeostasis. Curr Opin Cell Biol 33: 1-7, 2015.

14. Ideta T, Shirakami Y, Miyazaki T, Kochi T, Sakai H, Moriwaki H and Shimizu M: The dipeptidyl peptidase-4 inhibitor teneligliptin attenuates hepatic lipogenesis via AMPK activation in non-alcoholic fatty liver disease model mice. Int J Mol Sci 16: 29207-29218, 2015. 
15. Cheng S, Liang S, Liu Q, Deng Z, Zhang Y, Du J, Zhang Y, Li S Cheng B and Ling C: Diosgenin prevents high-fat diet-induced rat non-alcoholic fatty liver disease through the AMPK and LXR signaling pathways. Int J Mol Med 41: 1089-1095, 2018.

16. Smith BK, Marcinko K, Desjardins EM, Lally JS, Ford RJ and Steinberg GR: Treatment of nonalcoholic fatty liver disease: Role of AMPK. Am J Physiol Endocrinol Metab 311: E730-E740, 2016.

17. Wang ZQ, Zhang XH, Yu Y, Tipton RC, Raskin I, Ribnicky D, Johnson W and Cefalu WT: Artemisia scoparia extract attenuates non-alcoholic fatty liver disease in diet-induced obesity mice by enhancing hepatic insulin and AMPK signaling independently of FGF21 pathway. Metabolism 62: 1239-1249, 2013

18. Woods A, Williams JR, Muckett PJ, Mayer FV, Liljevald M, Bohlooly-Y $M$ and Carling D: Liver-specific activation of AMPK prevents steatosis on a high-fructose diet. Cell Rep 18: 3043-3051, 2017

19. Postic C, Dentin R, Denechaud P-D and Girard J: ChREBP, a transcriptional regulator of glucose and lipid metabolism. Annu Rev Nutr 27: 179-192, 2007.

20. Wang Y, Viscarra J, Kim S-J and Sul HS: Transcriptional regulation of hepatic lipogenesis. Nat Rev Mol Cell Biol 16: 678-689, 2015.

21. Saline M, Badertscher L, Wolter M, Lau R, Gunnarsson A, Jacso T, Norris T, Ottmann C, and Snijder A: AMPK and AKT protein kinases hierarchically phosphorylate the N-terminus of the FOXO1 transcription factor, modulating interactions with 14-3-3 proteins. J Biol Chem 294 (35):13106-13116, 2019 pii: jbc. RA119.008649.

22. Bandyopadhyay GK, Lu M, Avolio E, Siddiqui JA, Gayen JR, Wollam J, Vu CU, Chi NW, O'Connor DT and Mahata SK: Pancreastatin-dependent inflammatory signaling mediates obesity-induced insulin resistance. Diabetes 64: 104-116, 2015

23. Zhao L, Zhang C, Luo X, Wang P, Zhou W, Zhong S, Xie Y, Jiang Y, Yang P, Tang R, et al: CD36 palmitoylation disrupts free fatty acid metabolism and promotes tissue inflammation in non-alcoholic steatohepatitis. J Hepatol 69: 705-717, 2018.

24. Livak KJ and Schmittgen TD: Analysis of relative gene expression data using real-time quantitative PCR and the 2 (-Delta DeltaC (T)) Method. Methods 25: 402-408, 2001.

25. DeWeerdt S: Disease progression. Divergent paths. Nature 551: S92-S93, 2017.
26. Michelotti GA, Machado MV and Diehl AM: NAFLD, NASH and liver cancer. Nat Rev Gastroenterol Hepatol 10: 656-665, 2013.

27. Wong VW-S and Wong GL-H: A LEAN treatment for non-alcoholic steatohepatitis. Lancet 387: 628-630, 2016.

28. Kantor PF, Lucien A, Kozak R and Lopaschuk GD: The antianginal drug trimetazidine shifts cardiac energy metabolism from fatty acid oxidation to glucose oxidation by inhibiting mitochondrial long-chain 3-ketoacyl coenzyme A thiolase. Circ Res 86: 580-588, 2000.

29. Chen J, Lai J, Yang L, Ruan G, Chaugai S, Ning Q, Chen C and Wang DW: Trimetazidine prevents macrophage-mediated septic myocardial dysfunction via activation of the histone deacetylase sirtuin 1. Br J Pharmacol 173: 545-561, 2016.

30. Abdul-Wahed A, Guilmeau S and Postic C: Sweet sixteenth for ChREBP: Established roles and future goals. Cell Metab 26: 324-341, 2017.

31. Jump DB, Tripathy S and Depner CM: Fatty acid-regulated transcription factors in the liver. Annu Rev Nutr 33: 249-269, 2013.

32. Benhamed F, Denechaud P-D, Lemoine M, Robichon C, Moldes M, Bertrand-Michel J, Ratziu V, Serfaty L, Housset C, Capeau $\mathrm{J}$, et al: The lipogenic transcription factor ChREBP dissociates hepatic steatosis from insulin resistance in mice and humans. J Clin Invest 122: 2176-2194, 2012.

33. Zhang Y, Hu SL, Hu D, Jiang JG, Cui GL, Liu XD and Wang DW: miR-1322 regulates ChREBP expression via binding a 3'-UTR variant (rs1051943). J Cell Mol Med 22: 5322-5332, 2018.

34. Xie X, Yan D, Li H, Zhu Q, Li J, Fang YP, Cheung CW, Irwin MG, Xia Z and Lian Q: Enhancement of adiponectin ameliorates nonalcoholic fatty liver disease via inhibition of FoxO1 in Type I Diabetic Rats. J Diabetes Res 2018: 6254340, 2018.

35. O-Sullivan IS, Zhang W, Wasserman DH, Liew CW, Liu J, Paik J, DePinho RA, Stolz DB, Kahn CR, Schwartz MW, et al: FoxO1 integrates direct and indirect effects of insulin on hepatic glucose production and glucose utilization. Nat Commun 6: 7079, 2015.

This work is licensed under a Creative Commons Attribution-NonCommercial-NoDerivatives 4.0 International (CC BY-NC-ND 4.0) License. 